\title{
Phenomenological study of spin-triplet ferromagnetic superconductors
}

\author{
D.V.Shopova, T.E.Tsvetkov, D.I.Uzunov \\ CP Laboratory, Institute of Solid State Physics, \\ Bulgarian Academy of Sciences, \\ BG-1784 Sofia, Bulgaria
}

Received November 15, 2004

\begin{abstract}
Unconventional superconductivity with spin-triplet Cooper pairing is reviewed based on the quasi-phenomenological Ginzburg-Landau theory. The superconductivity, in particular, the mixed phase of coexistence of ferromagnetizm and unconventional superconductivity is triggered by the spontaneous magnetization. The mixed phase is stable whereas the other superconducting phases that usually exist in unconventional superconductors are either unstable, or, for particular values of the parameters of the theory, some of these phases are metastable at relatively low temperatures in a quite narrow domain of the phase diagram. The phase transitions from the normal phase to the phase of coexistence is of first order while the phase transition from the ferromagnetic phase to the coexistence phase can be either of first or second order depending on the concrete substance. The Cooper pair and crystal anisotropy are relevant to a more precise outline of the phase diagram shape and reduce the degeneration of the ground states of the system but they do not drastically effect the phase stability domains and the thermodynamic properties of the respective phases.
\end{abstract}

Key words: superconductivity, ferromagnetizm, phase diagram, order parameter profile, anisotropy

PACS: 74.20.De, 74.20.Rp

\section{Inroduction}

The formation of Cooper pairs with a nonzero angular momentum was theoretically predicted [1] in 1959 as a mechanism of superfluidity of Fermi liquids. In 1972 the same phenomenon - unconventional superfluidity due to a $p$-wave (spin triplet) Cooper pairing of ${ }^{3} \mathrm{He}$ atoms, was experimentally discovered in the $\mathrm{mK}$ range of temperatures; for details and theoretical description, see [2-4]. In contrast to the standard $s$-wave pairing in usual (conventional) superconductors, where the electron pairs are formed by an attractive electron-electron interaction due to a virtual 
phonon exchange, the widely accepted mechanism of the Cooper pairing in superfluid ${ }^{3} \mathrm{He}$ is based on an attractive interaction between the fermions ( ${ }^{3} \mathrm{He}$ atoms) due to a virtual exchange of spin fluctuations. Certain spin fluctuation mechanisms of unconventional Cooper pairing of electrons were also proposed for the depiction of heavy fermion superconductors discovered in 1979 (see, e. g., [5-7]) as well as for some classes of high-temperature superconductors (see, e. g., [8-16]).

The possible superconducting phases in unconventional superconductors are described in the framework of the general Ginzburg-Landau (GL) effective free energy functional [13] using the symmetry groups theory. A variety of possible superconducting orderings were predicted for different crystal structures [17-22]. A detailed thermodynamic analysis $[11,18]$ of the homogeneous (Meissner) phases and a renormalization group investigation [11] of the superconducting phase transition up to the two-loop approximation have been also performed (for a three-loop renormalization group analysis, see [23]; for effects of magnetic fluctuations and disorder, see [24,25]).

In 2000, experiments [26] at low temperatures $(T \approx 1 \mathrm{~K})$ and high pressure $(T \approx 1 \mathrm{GPa})$ demonstrated the existence of spin triplet superconducting states in the metallic compound $\mathrm{UGe}_{2}$. This superconductivity is triggered by spontaneous magnetization of the ferromagnetic phase which exists at much higher temperatures and coexists with the superconducting phase in the whole domain of existence of the latter below $T \approx 1 \mathrm{~K}$; see also experiments published in $[27,28]$, and the discussion in [29]. Moreover, the same phenomenon of existence of superconductivity at low temperatures and high pressure in the domain of the $(T, P)$ phase diagram where the ferromagnetic order is present has been observed in other ferromagnetic metallic compounds $\left(\mathrm{ZrZn}_{2}\right.$ [30] and URhGe [31]) soon after the discovery [26] of superconductivity in $\mathrm{UGe}_{2}$.

In contrast to other superconducting materials, for example, ternary and Chevrel phase compounds, where the effects of magnetic order on superconductivity are also substantial (see, e. g., [32-35]), in these ferromagnetic compounds the phase transition temperature $\left(T_{\mathrm{f}}\right)$ to the ferromagnetic state is much higher than the phase transition temperature $\left(T_{\mathrm{FS}}\right)$ from ferromagnetic to a (mixed) state of coexistence of ferromagnetizm and superconductivity. For example, in $\mathrm{UGe}_{2} T_{\mathrm{FS}}$ is $0.8 \mathrm{~K}$ whereas the critical temperature of the phase transition from paramagnetic to ferromagnetic state in the same material is $T_{\mathrm{f}}=35 \mathrm{~K}[26,27]$. It can be supposed that in such substances the material parameter $T_{\mathrm{s}}$ defined as the (usual) critical temperature of the second order phase transition from normal to uniform (Meissner) supercondicting state in a zero external magnetic field is much lower than the phase transition temperature $T_{\mathrm{FS}}$. Note, that the mentioned experiments with the compounds $\mathrm{UGe}_{2}$, URhGe, and $\mathrm{ZrZn}_{2}$ do not give any evidence for the existence of a standard normalto-superconducting phase transition in a zero external magnetic field.

Moreover, it seems that the superconductivity in the metallic compounds, mentioned above, always coexists with the ferromagnetic order and is enhanced by it. As claimed in [26] in these systems the superconductivity seems to arise from the same electrons that create the band magnetism, and is most naturally understood as a triplet rather than spin-singlet pairing phenomenon. Note, that all three metallic 
compounds, mentioned so far, are itinerant ferromagnets. A similar type of unconventional superconductivity has been suggested [36] as a possible outcome of recent experiments in Fe [37], where a superconducting phase is discovered at temperatures below $2 \mathrm{~K}$ for pressures between 15 and $30 \mathrm{GPa}$. Note, that both vortex and Meissner superconductivity phases [37] have been found in the high-pressure crystal modification of Fe which has a hexagonal close-packed crystal structure. In this hexagonal lattice the strong ferromagnetizm of the usual bcc iron crystal probably disappears [36]. Thus one can hardly claim that there is a coexistence of ferromagnetizm and superconductivity in Fe but the clear evidence of a superconductivity is also a remarkable achievement.

The important point in all discussions of the interplay between superconductivity and ferromagnetizm is that a small amount of magnetic impurities can destroy superconductivity in conventional ( $s$-wave) superconductors by breaking up the $(s-$ wave) electron pairs with opposite spins (paramagnetic impurity effect [38]). In this aspect the phenomenological arguments [39] and the conclusions based on the microscopic theory of magnetic impurities in $s$-wave superconductors [38] are in a complete agreement with each other; see, e. g., [32-35]. In fact, a total suppression of conventional ( $s$-wave) superconductivity should occur in the presence of an uniform spontaneous magnetization $\boldsymbol{M}$, i.e., in a standard ferromagnetic phase [39]. The physical reason for this suppression is the same as in the case of magnetic impurities, namely, the opposite electron spins in the $s$-wave Cooper pair turn along the vector $\boldsymbol{M}$ in order to lower their Zeeman energy and, hence, the pairs break down. Therefore, the ferromagnetic order can hardly coexist with conventional superconducting states. Especially this is so for the coexistence of uniform superconducting and ferromagnetic states when the superconducting order parameter $\psi(\boldsymbol{x})$ and the magnetization $\boldsymbol{M}(\boldsymbol{x})$ do not depend on the spatial vector $\boldsymbol{x}$.

However, a coexistence of $s$-wave superconductivity and ferromagnetizm may appear in uncommon materials and under quite special circumstances. Furthermore, let us emphasize that the conditions for the coexistence of nonuniform ("vertex", "spiral", "spin-sinosoidal" or "helical") superconducting and ferromagnetic states are less restrictive than those for the coexistence of uniform superconducting and ferromagnetic orders. Coexistence of nonuniform phases has been discussed in detail, in particular, in the experiment and theory of ternary and Chevrel-phase compounds, where such coexistence seems quite likely; for a comprehensive review, see, for example, [32-35,40].

In fact, the only two superconducting systems for which the experimental data allow assumptions in favor of a coexistence of superconductivity and ferromagnetizm are the rare earth ternary boride compound $\mathrm{ErRh}_{4} \mathrm{~B}_{4}$ and the Chervel phase compound $\mathrm{HoMo}_{6} \mathrm{~S}_{8}$; for a more extended review, see [33,41]. In these compounds the phase of coexistence most likely appears in a very narrow temperature region just below the Curie temperature $T_{\mathrm{f}}$ of the ferromagnetic phase transition. At lower temperatures the magnetic moments of the rare earth $4 f$ electrons become better aligned, the magnetization increases and the $s$-wave superconductivity pairs formed by the conduction electrons disintegrate. 
We shall not extend our consideration over all important aspects of the long standing problem of coexistence of superconductivity and ferromagnetizm. We shall rather concentrate on the description of the newly discovered coexistence of ferromagnetizm and unconventional (spin-triplet) superconductivity in the itinerant ferromagnets $\mathrm{UGe}_{2}, \mathrm{ZrZn}_{2}$, and URhGe. Here we wish to emphasize that the main object of our discussion is the superconductivity of these compounds and, in a second place in the rate of importance we put the problem of coexistence. The reason is that the existence of superconductivity in such itinerant ferromagnets is a highly nontrivial phenomenon. As noted in [42] the superconductivity in these materials seems difficult to be explained in terms of previous theories [32,33,35] and requires new concepts for the interpretation of experimental data.

We have already mentioned that in ternary compounds the ferromagnetizm comes from the localized $4 f$ electrons whereas the s-wave Cooper pairs are formed by conduction electrons. In $\mathrm{UGe}_{2}$ and $\mathrm{URhGe}$ the $5 f$ electrons of $\mathrm{U}$ atoms form both superconducting and ferromagnetic order [26,31]. In $\mathrm{ZrZn}_{2}$ the same twofold role is played by the $4 d$ electrons of Zr. Therefore the task is to describe this behavior of the band electrons at a microscopic level. One may speculate about a spin-fluctuation mediated unconventional Cooper pairing as is in case of ${ }^{3} \mathrm{He}$ and heavy fermion superconductors. These important issues do not have yet a reliable answer and for this reason we shall confine our consideration to a phenomenological level.

Reliable experimental data, for example, the data on the coherence length and the superconducting gap $[26,27,31,30]$, are in favor of the conclusion about a spintriplet Cooper pairing in these metallic compounds, although the mechanism of the pairing remains unclear. We shall essentially use this reliable conclusion. Besides, this point of view is consistent with the experimental observation of coexistence of superconductivity only in the low temperature part of the ferromagnetic domain of the phase diagram $(T, P)$ which means that a pure (non ferromagnetic) superconducting phase has not been observed. This circumstance is also in favor of the assumption of a spin-triplet superconductivity. Our investigation leads to the results which confirm this general picture.

Based on the experimental data and on the conclusions presented for the first time in $[26,29]$ and shortly afterwards confirmed in $[27,28,30,31]$ it can be accepted that the superconductivity in these magnetic compounds is considerably enhanced by the ferromagnetic order parameter $\boldsymbol{M}$ and, perhaps, it could not exist without this "mechanism of ferromagnetic trigger", or, in short, " $\boldsymbol{M}$-trigger". Such a phenomenon is possible for spin-triplet Cooper pairs, where the electron spins point parallel to each other and their turning along the vector of spontaneous magnetization $\boldsymbol{M}$ does not cause a breakdown of the spin-triplet Cooper pairs but rather stabilizes them and, perhaps, stimulates their creation. We shall describe this phenomenon at a phenomenological level.

Recently, the phenomenological theory that explains the coexistence of ferromagnetizm and unconventional spin-triplet superconductivity of GL type was developed [42,43]. The possible low-order couplings between superconducting and ferromagnetic order parameters were derived by means of general symmetry group 
arguments. Thus, several important features of the superconducting vortex state in the ferromagnetic phase of unconventional ferromagnetic superconductors were established $[42,43]$.

In this paper we shall use the approach presented in $[42,43]$ to investigate the conditions for the occurrence of the Meissner phase and to demonstrate that the presence of ferromagnetic order enhances the $p$-wave superconductivity. Our consideration is focused on the ground state, namely, we are interested in uniform phases, where the order parameters (the superconducting order parameter $\psi$ and the magnetization vector $M=\left\{M_{j}, j=1,2,3\right\}$ ), do not depend on the spatial vector $\vec{x} \in V$, where $V$ is the volume of the system. Recent results regarding the phase diagram and the phase transitions $[44,45]$, and thermodynamic quantities [46] will be essentially used in our investigation.

Our study is based on the mean-field approximation [13] as well as on familiar results for the possible phases in nonmagnetic superconductors with triplet ( $p$-wave) Cooper pairs $[11,12,18]$. Results from [44-47] will be reviewed and extended. In our proceeding investigation [44-46] both Cooper pair anisotropy and crystal anisotropy have been neglected in order to clarify the main effect of the coupling between the ferromagnetic and superconducting order parameters. The phenomenological GL free energy is quite complex and the inclusion of these anisotropies is related with lengthy formulae and a multivariant analysis which obscures the final results. Here we shall pay our attention to the effect of the Cooper pairs anisotropy.

There exists a formal similarity between the phase diagram we have obtained and the phase diagram of certain improper ferroelectrics [48-53]. The variants of the theory of improper ferroelectrics, known before 1980, were criticized in [53] for their oversimplification and inconsistency with the experimental results. But the further development of the theory has no such disadvantages (see, e. g., [51,52]). We should emphasize that the symmetry of the GL model of spin-triplet ferromagnetic superconductors is quite different from the symmetry of the known models in ferroelectrics and, hence, the results for ferroelectric systems can hardly be applied to superconductors without additional investigations.

\section{Ginzburg-Landau free energy}

Consider the GL free energy $F(\psi, \boldsymbol{M})=V f(\psi, \boldsymbol{M})$, where the free energy density $f(\psi, \boldsymbol{M})$ (for short hereafter called "free energy") of a spin-triplet ferromagnetic superconductor is given by

$$
\begin{aligned}
f(\psi, \boldsymbol{M})= & a_{\mathrm{s}}|\psi|^{2}+\frac{b_{\mathrm{s}}}{2}|\psi|^{4}+\frac{u_{\mathrm{s}}}{2}\left|\psi^{2}\right|^{2}+\frac{v_{\mathrm{s}}}{2} \sum_{j=1}^{3}\left|\psi_{j}\right|^{4}+a_{\mathrm{f}} \boldsymbol{M}^{2}+\frac{b_{\mathrm{f}}}{2} \boldsymbol{M}^{4} \\
& +\mathrm{i} \gamma_{0} \boldsymbol{M} \cdot\left(\psi \times \psi^{*}\right)+\delta \boldsymbol{M}^{2}|\psi|^{2} .
\end{aligned}
$$

In (1), $\psi=\left\{\psi_{j} ; j=1,2,3\right\}$ is a three-dimensional complex vector $\left(\psi_{j}=\psi_{j}^{\prime}+\mathrm{i} \psi_{j}^{\prime \prime}\right)$ describing the unconventional (spin-triplet) superconducting order and $\boldsymbol{B}=(\boldsymbol{H}+$ $4 \pi \boldsymbol{M})=\nabla \times \boldsymbol{A}$ is the magnetic induction; $\boldsymbol{H}=\left\{H_{j} ; j=1,2,3\right\}$ is the external 
magnetic field, $\boldsymbol{A}=\left\{A_{j} ; j=1,2,3\right\}$ is the magnetic vector potential $(\nabla \cdot A=0)$. In $(1), b_{\mathrm{s}}>0, b_{\mathrm{f}}>0, a_{\mathrm{f}}=\alpha_{\mathrm{f}}\left(T-T_{\mathrm{f}}\right)$ is given by the positive material parameter $\alpha_{\mathrm{f}}$ and the ferromagnetic critical temperature $T_{\mathrm{f}}$ corresponding to a simple ferromagnet $(\psi \equiv 0)$, and $a_{\mathrm{s}}=\alpha_{\mathrm{s}}\left(T-T_{\mathrm{s}}\right)$, where $\alpha_{\mathrm{s}}$ is another positive material parameter and $T_{\mathrm{s}}$ is the critical temperature of a standard second order phase transition which may occur at $|H|=\mathcal{M}=0 ; \mathcal{M}=|\boldsymbol{M}|$. The parameter $u_{\mathrm{s}}$ describes the anisotropy of the spin-triplet Cooper pair whereas the crystal anisotropy is described by the parameter $v_{\mathrm{s}}[11,18]$.

The two orders - the magnetization vector $M=\left\{M_{j}\right\}$ and $\psi=\left\{A_{j}\right\}$, interact through the last two terms in (1). The $\gamma_{0}$-term [43] ensures the triggering of the superconductivity by the ferromagnetic order $\left(\gamma_{0}>0\right)$ whereas the $\delta$-term makes the model more realistic in the strong coupling limit [42]. Both $\psi M$-interaction terms included in (1) are important for a correct description of the temperature-pressure $(T, P)$ phase diagram of the ferromagnetic superconductor $[44,45]$. In general, the parameter $\delta$ for ferromagnetic superconductors may take both positive and negative values.

As we are interested in the ground state properties, we set the external magnetic field equal to zero $(H=0)$. Besides, we emphasize that the magnetization vector $M$ may produce vortex superconducting phase in case of type II superconductivity. The investigation of nonuniform (vortex) states can be made with the help of gradient terms in the free energy which take into account the spatial variations of the order parameter field $\psi$. This task is beyond our present consideration. Rather we investigate the basic problem about the possible stable uniform (Meissner) superconducting phases which may coexist with uniform ferromagnetic order. For this aim the free energy (1) is quite convenient.

In case of a strong easy axis type of magnetic anisotropy, as is in $\mathrm{UGe}_{2}$ [26], the overall complexity of mean-field analysis of the free energy $f(\psi, \boldsymbol{M})$ can be avoided by performing an "Ising-like" description: $\boldsymbol{M}=(0,0, \mathcal{M})$. Further, because of the equivalence of the "up" and "down" physical states $( \pm \boldsymbol{M})$ the thermodynamic analysis can be done within the "gauge" $\mathcal{M} \geqslant 0$. But this stage of consideration can also be achieved without the help of crystal anisotropy arguments. When the magnetic order has a continuous symmetry one may take advantage of the symmetry of the total free energy $f(\psi, \boldsymbol{M})$ and avoid the study of equivalent thermodynamic states that occur as a result of the respective symmetry breaking at the phase transition point but they have no effect on thermodynamics of the system. In the isotropic system one may again choose a gauge, in which the magnetization vector has the same direction as $z$-axis $\left(|\boldsymbol{M}|=M_{z}=\mathcal{M}\right)$ and this will not influence the generality of thermodynamic analysis. Here we shall prefer the alternative description within which the ferromagnetic state may occur as two equivalent "up" and "down" domains with magnetizations $\mathcal{M}$ and $-\mathcal{M}$, respectively.

We shall use adequate notations which reduce the number of parameters. With the help of

$$
b=\left(b_{\mathrm{s}}+u_{\mathrm{s}}+v_{\mathrm{s}}\right)>0
$$


we redefine the order parameters and the other parameters in the following way:

$$
\begin{aligned}
& \varphi_{j}=b^{1 / 4} \psi_{j}=\phi_{j} \mathrm{e}^{\theta_{j}}, \quad M=b_{\mathrm{f}}^{1 / 4} \mathcal{M}, \\
& r=\frac{a_{\mathrm{s}}}{\sqrt{b}}, \quad t=\frac{a_{\mathrm{f}}}{\sqrt{b_{\mathrm{f}}}}, \quad w=\frac{u_{\mathrm{s}}}{b}, \quad v=\frac{v_{\mathrm{s}}}{b}, \\
& \gamma=\frac{\gamma_{0}}{b^{1 / 2} b_{\mathrm{f}}^{1 / 4}}, \quad \gamma_{1}=\frac{\delta}{\left(b b_{\mathrm{f}}\right)^{1 / 2}} .
\end{aligned}
$$

Having in mind our approximation of uniform $\psi$ and $\boldsymbol{M}$ and the notations (2)(3), the free energy density $f(\psi, M)$ can be written in the form

$$
\begin{aligned}
f(\psi, M)= & r \phi^{2}+\frac{1}{2} \phi^{4}+2 \gamma \phi_{1} \phi_{2} M \sin \left(\theta_{2}-\theta_{1}\right)+\gamma_{1} \phi^{2} M^{2}+t M^{2}+\frac{1}{2} M^{4} \\
& -2 w\left[\phi_{1}^{2} \phi_{2}^{2} \sin ^{2}\left(\theta_{2}-\theta_{1}\right)+\phi_{1}^{2} \phi_{3}^{2} \sin ^{2}\left(\theta_{1}-\theta_{3}\right)+\phi_{2}^{2} \phi_{3}^{2} \sin ^{2}\left(\theta_{2}-\theta_{3}\right)\right] \\
& -v\left[\phi_{1}^{2} \phi_{2}^{2}+\phi_{1}^{2} \phi_{3}^{2}+\phi_{2}^{2} \phi_{3}^{2}\right] .
\end{aligned}
$$

In the above free energy the order parameters $\psi$ and $\boldsymbol{M}$ are defined per unit volume.

We assume that $T_{\mathrm{f}}>T_{\mathrm{s}}$. This is the case when the superconductivity is triggered by the magnetic order. We shall discuss the stable phases in the temperature region $T>T_{\mathrm{s}}$. The case $T_{\mathrm{f}}<T_{\mathrm{s}}$ may be also important for neutron stars so it needs a special investigation. When $T_{\mathrm{s}} \sim T_{\mathrm{f}}$ a quite simple analytical treatment is possible. All these cases may be of interest to the description of ferromagnetic superconductivity in stellar objects whereas in condensed matter only the case of $T_{\mathrm{f}} \gg T_{\mathrm{s}}$ has been observed so far.

We work in the framework of the standard mean-field analysis [13]. The stable phases correspond to global minima of the GL energy (1). The equilibrium phase transition line separating two phases is defined by thermodynamic states, where the respective GL free energies are equal.

\section{Phases}

We shall not enumerate and discuss all phases described by (1). Rather we shall focus our attention on the stable phases at relatively high temperatures $\left(T>T_{\mathrm{s}}\right)$. The calculations show that for temperatures $T>T_{\mathrm{s}}$, i.e., for $r>0$, there are three stable phases. Two of them are quite simple: the normal phase $(\psi=M=0)$ with existence and stability domains given by $t>0$ and $r>0$, and the ferromagnetic phase (FM) given by $\psi=0$ and $M^{2}=-t$ whose existence condition is $t<0$, there is a stability domain defined by the inequalities $r>\gamma_{1} t$ and

$$
r>\gamma_{1} t+\gamma \sqrt{-t}
$$

The third stable phase is the phase of coexistence of superconductivity and ferromagnetizm (hereafter referred to as FS). This phase is the main object of our 
consideration. It is given by the following equations:

$$
\begin{aligned}
& \phi_{1}=\phi_{2}=\frac{\phi}{\sqrt{2}}, \quad \phi_{3}=0 \\
& \phi^{2}=\left( \pm \gamma M-r-\gamma_{1} M^{2}\right) \\
& \left(1-\gamma_{1}^{2}\right) M^{3} \pm \frac{3}{2} \gamma \gamma_{1} M^{2}+\left(t-\frac{\gamma^{2}}{2}-\gamma_{1} r\right) M \pm \frac{\gamma r}{2}=0
\end{aligned}
$$

and

$$
\left(\theta_{2}-\theta_{1}\right)=\mp \frac{\pi}{2}+2 \pi k
$$

$(k=0, \pm 1, \ldots)$. The upper sign in $(7)-(9)$ corresponds to a domain where $\sin \left(\theta_{2}-\right.$ $\left.\theta_{1}\right)=-1$ and the lower sign corresponds to a second domain which may be referred to as $\mathrm{FS}^{*}$; there $\sin \left(\theta_{2}-\theta_{1}\right)=1$. These two domains are equivalent and describe the same ordering. We shall focus on the upper sign in (7)-(9), i.e. on FS. The phase diagram $(t, r)$ has qualitatively the same shape as the phase transition lines corresponding to $w=0$ but there are essential quantitative differences between them. We shall discuss them in the next section. Note, that the system exhibits both first and second order phase transitions and complex phase transition points: triple and tricritical points $[44,47]$.

\section{Anisotropy effects}

Our analysis demonstrates that when the anisotropy of the Cooper pairs is taken into account, there will not be drastic changes in the shape of the phase diagram for $r>0$ and the order of the respective phase transitions. Of course, there will be some changes in the size of the phase domains and the formulae for the thermodynamic quantities. Besides, and this seems to be the main anisotropy effect, the $w$ - and $v$-terms in the free energy lead to a stabilization of the order along the main crystal directions which, in other words, means that the degeneration of the possible ground states is considerably reduced. So there will be a smaller number of marginally stable states.

Let us neglect the crystal anisotropy by setting $v_{\mathrm{s}}=0$ in (1) and concentrate our attention on the Cooper pair anisotropy described by the $u_{\mathrm{s}}$-term in the GL model. The dimensionless anisotropy parameter $w \sim u_{\mathrm{s}}$ given by (3) can be either positive or negative depending on the sign of $u_{\mathrm{s}}$. Obviously when $u_{\mathrm{s}}>0$, the parameter $w$ will be positive too $(0<w<1)$. We shall illustrate the effect of Cooper-pair anisotropy in this case. The order parameters $\left(M, \phi_{j}, \theta_{j}\right)$ are given by $(6),(9)$,

$$
\phi^{2}=\frac{ \pm \gamma M-r-\gamma_{1} M^{2}}{(1-w)} \geqslant 0
$$

and

$$
\left(1-w-\gamma_{1}^{2}\right) M^{3} \pm \frac{3}{2} \gamma \gamma_{1} M^{2}+\left[t(1-w)-\frac{\gamma^{2}}{2}-\gamma_{1} r\right] M \pm \frac{\gamma r}{2}=0
$$


where the meaning of the upper and lower sign is the same as explained just below (9). We consider the FS domain corresponding to the upper sign in the (10) and (11). The stability conditions for FS read,

$$
\begin{aligned}
\frac{(2-w) \gamma M-r-\gamma_{1} M^{2}}{1-w} & \geqslant 0, \\
\gamma M-w r-w \gamma_{1} M^{2} & \geqslant 0,
\end{aligned}
$$

and

$$
\frac{1}{1-w}\left[3\left(1-w-\gamma_{1}^{2}\right) M^{2}+3 \gamma \gamma_{1} M+t(1-w)-\frac{\gamma^{2}}{2}-\gamma_{1} r\right] \geqslant 0 .
$$

For $M \neq\left(\gamma / 2 \gamma_{1}\right)$ we can express the function $r(M, t)$ defined by (11), substitute the obtained expression for $r(M, t)$ in the existence (10) and stability conditions (12)(14) and do the analysis in the same way as for $w=0$ [47]. The most substantial qualitative difference between the cases $w>0$ and $w<0$ is that for $w<0$ the stability of FS is bounded for $r<0$.

\section{Conclusion}

We have done an investigation of the M-trigger effect in unconventional ferromagnetic superconductors. This effect due to the $M \psi_{1} \psi_{2}$-coupling term in the GL free energy consists in bringing into existence the superconductivity in a domain of the phase diagram of the system that is entirely in the region of existence of the ferromagnetic phase. This form of coexistence of unconventional superconductivity and ferromagnetic order is possible for temperatures above and below the critical temperature $T_{\mathrm{s}}$, which corresponds to the standard phase transition of second order from normal to Meissner phase, i. e., a usual uniform superconductivity in a zero external magnetic field, which appears outside the domain of existence of the ferromagnetic order. Our investigation has been mainly intended to clarify the thermodynamic behaviour at temperatures $T_{\mathrm{s}}<T<T_{\mathrm{f}}$, where the superconductivity cannot appear without the mechanism of M-triggering. We have described the possible ordered phases (FM and FS) in this most interesting temperature interval.

The Cooper pair and crystal anisotropies have also been investigated and their main effects on the thermodynamics of the triggered phase of coexistence are established. In discussions of concrete real materials one should take into account the respective crystal symmetry but the variation of the essential thermodynamic properties with the change of the type of symmetry is not substantial when the low symmetry and low order (in both $M$ and $\psi$ ) $\gamma$-term is present in the free energy.

Below the superconducting critical temperature $T_{\mathrm{s}}$ a variety of pure superconducting and mixed phases of coexistence of superconductivity and ferromagnetizm exists and the thermodynamic behavior at these relatively low temperatures is more complex than in the known cases of improper ferroelectrics. The case $T_{\mathrm{f}}<T_{\mathrm{s}}$ also needs a special investigation. Our results are referred to the possible uniform superconducting and ferromagnetic states. Vortex and other nonuniform phases need a separate study. 
The relation of the present investigation to the properties of real ferromagnetic compounds, such as $\mathrm{UGe}_{2}$, URhGe, and $\mathrm{ZrZn}_{2}$, has been discussed throughout the text. In these real compounds the ferromagnetic critical temperature is much larger than the superconducting critical temperature $\left(T_{\mathrm{f}} \gg T_{\mathrm{s}}\right)$ and that is why the M-triggering of the spin-triplet superconductivity is very strong. Moreover, the $\gamma_{1^{-}}$ term is important to stabilize the FM order up to the absolute zero $(0 \mathrm{~K})$, as is in the known spin-triplet ferromagnetic superconductors. The neglect [43] of the symmetry conserving $\gamma_{1}$-term hinders the proper description of real substances of this type. More experimental information about the values of the material parameters $\left(a_{\mathrm{s}}, a_{\mathrm{f}}, \ldots\right)$ is required in order to outline the thermodynamic behavior and the phase diagram in terms of thermodynamic parameters $T$ and $P$. In particular, a reliable knowledge about the dependence of the parameters $a_{\mathrm{s}}$ and $a_{\mathrm{f}}$ on the pressure $P$, on the value of the characteristic temperature $T_{\mathrm{s}}$ and on the ratio $a_{\mathrm{s}} / a_{\mathrm{f}}$ at zero temperature are of primary interest.

\section{Acknowledgements}

The authors warmly greet Reinhard Folk with his 60th anniversary! D.V.S. and D.I.U. thank Reinhard Folk for the valuable collaboration, discussions, and professional support over the last fifteen years.

\section{References}

1. Pitaevskii L.P., Zh. Eksp. Teor. Fiz., 1959, 37, 1794 (in Russian); Sov. Phys. JETP, 1960, 10, 1267 (in English).

2. Leggett A.J., Rev. Mod. Phys., 1975, 47, 331.

3. Vollhardt D., P. Wölfle P.W. The Superfluid Phases of Helium 3. Taylor and Francis, London, 1990.

4. Volovik G.E. The Universe in a Helium Droplet. Oxford University Press, Oxford, 2003.

5. Stewart G.R., Rev. Mod. Phys., 1984, 56, 755.

6. Sigrist M., Ueda K., Rev. Mod. Phys., 1991, 63, 239.

7. Mineev V.P., Samokhin K.V., Introduction to Unconventional Superconductivity. Gordon and Breach, Amsterdam, 1999.

8. Sigrist M., Ruce T.M., Z. Phys. B. - Condensed Matter, 1987, 68, 9.

9. Annett J.A., Randeria M., Renn S.R., Phys. Rev. B, 1988, 3, 4660.

10. Volovik G.E., JETP Lett., 1988, 48, 41;

Pis'ma Zh. Eksp. Teor. Fiz., 1988, 48, 39.

11. Blagoeva E.J., Busiello G., De Cesare L., Millev Y.T., Rabuffo I., Uzunov D.I., Phys. Rev. B, 1990, 42, 6124.

12. Uzunov D.I. Phenomenological studies of unconventional superconductors. Advances in Theoretical Physics, edited by E.Caianiello, p. 96. World Scientific, Singapore, 1991.

13. Uzunov D.I. Theory of Critical Phenomena. World Scientific, Singapore, 1993.

14. Annett J.A., Contemp. Physics, 1995, 36, 323.

15. Van Harlingen D.J., Rev. Mod. Phys., 1995, 67, 515. 
16. Tsuei C.C., Kirtly J.R., Rev. Mod. Phys., 2000, 72, 969.

17. Volovik G.E., Gor'kov L.P., JETP Lett., 1984, 39, 674.;

Pis'ma Zh. Eksp. Teor. Fiz., 1984, 39, 550.

18. Volovik G.E., Gor'kov L.P., Sov. Phys. JETP, 1985, 61, 843.;

Zh. Eksp. Teor. Fiz., 1985, 88, 1412.

19. Ueda K., Rice T.M., Phys. Rev. B, 1985, 31, 7114.

20. Blount E.I., Phys. Rev. B, 1985, 32, 2935.

21. Ozaki M., Machida K., Ohmi T., Progr. Theor. Phys., 1985, 74, 221.

22. Ozaki M., Machida K., Ohmi T., Progr. Theor. Phys., 1986, 75, 442.

23. Antonenko S.A., Sokolov A.I., Phys. Rev. B, 1994, 94, 15901.

24. Busiello G., de Cesare L., Millev Y.T., Rabuffo I., Uzunov D.I., Phys. Rev. B, 1991, 43, 1150.

25. Busiello G., Uzunov D.I., Phys. Rev. B, 1990, 42, 1018.

26. Saxena S.S., Agarwal P., Ahilan K., Grosche F.M., Haselwimmer R.K.W., Steiner M.J., Pugh E., Walker I.R., Julian S.R., Monthoux P., Lonzarich G.G., Huxley A., Sheikin I., Braithwaite D., Flouquet J., Nature, 2000, 406, 587.

27. Huxley A., Sheikin I., Ressouche E., Kernavanois N., Braithwaite D., Calemczuk R., Flouquet J., Phys. Rev. B, 2001, 63, 144519-1.

28. Tateiwa N., Kobayashi T.C., Hanazono K., Amaya A., Haga Y., Settai R., Onuki Y., J. Phys. Condensed Matter, 2001, 13, L17.

29. Coleman P., Nature, 2000, 406, 580.

30. Pfleiderer C., Uhlatz M., Hayden S.M., Vollmer R., Löhneysen H.V., Berhoeft N.R., Lonzarich G.G., Nature, 2001, 412, 58.

31. Aoki D., Huxley A., Ressouche E., Braithwaite D., Flouquet J., Brison J.-P., Lhotel E., Paulsen C., Nature, 2001, 413, 613.

32. Vonsovsky S.V., Izyumov Yu.A, Kurmaev E.Z. Superconductivity of Transition Metals. Springer Verlag, Berlin, 1982.

33. Maple M.B., Fisher F. (eds). Superconductivity in Ternary Compounds, Parts I and II. Springer Verlag, Berlin, 1982.

34. Sinha S.K. Superconductivity in Magnetic and Exotic Materials, ed. Matsubara T., Kotani A. Springer Verlag, Berlin, 1984.

35. Kotani A. Superconductivity in Magnetic and Exotic Materials, ed. Matsubara T., Kotani A. Springer Verlag, Berlin, 1984.

36. Saxena S.S., Littlewood P.B., Nature, 2001, 412, 290.

37. Shimizu K., Kikura T., Furomoto S., Takeda K., Kontani K., Onuki Y., Amaya K., Nature, 2001, 412, 316.

38. Abrikosov A.A., Gor'kov L.P., Zh. Eksp. Teor. Fiz., 1960, 39, 1781;

Sov. Phys. JETP, 1961, 12, 1243.

39. Ginzburg V.L., Zh. Eksp. Teor. Fiz., 1956, 31, 202;

Sov. Phys. JETP, 1957, 4, 153.

40. Buzdin A.I., Bulaevskii L.N., Krotov S.S., Zh. Eksp. Teor. Fiz., 1983, 85, 678;

Sov. Phys. JETP, 1983, 58, 395.

41. Machida K., Nakanishi H., Phys. Rev. B, 1984, 30, 122.

42. Machida K., Ohmi T., Phys. Rev. Lett., 2001, 86, 850.

43. Walker M.B., Samokhin K.V., Phys. Rev. Lett., 2002, 88, 207001-1.

44. Shopova D.V., Uzunov D.I., Phys. Lett. A, 2003, 313, 139.

45. Shopova D.V., Uzunov D.I., J. Phys. Studies, 2003, 7, 426. 
46. Shopova D.V., Uzunov D.I., Compt. Rend. Acad. Bulg. Sciences, 2003, 56, 35.

47. Shopova D.V., Uzunov D.I. Phases and phase transitions in spin-triplet ferromagnetic superconductors. Progress in Ferromagnetism Research. Nova Publishers, New York, 2004.

48. Gufan Yu.M., Torgashev V.I., Sov. Phys. Solid State, 1980, 22, 951;

Fiz. Tv. Tela, 1980, 22, 1629.

49. Gufan Yu.M., Torgashev V.I., Sov. Phys. Solid State, 1981, 23, 1129.

50. Latush L.T., Torgashev V.I., Smutny F., Ferroelectrics Letts., 1985, 4, 37.

51. Tolédano J-C., Tolédano P. The Landau Theory of Phase Transitions. World Scientific, Singapore, 1987.

52. Gufan Yu.M. Structural Phase Transitions. Nauka, Moskow, 1982 (in Russian).

53. Cowley R.A., Adv. Phys., 1980, 29, 1.

\title{
Феноменологічне дослідження спін-триплетних Феромагнітних надпровідників
}

\author{
Д.В.Шопова, Т.Є.Цвєтков, Д.І.Узунов \\ СР лабораторія, Інститут фізики твердого стану, \\ Болгарська академія наук, \\ BG-1784 Софія, Болгарія
}

Отримано 15 листопада 2004 р.

Оглянуто нетрадиційну надпровідність із спін-триплетним куперівським спарюванням, базуючись на квазі-феноменологічній теорії Гінзбурга-Ландау. Надпровідність, а зокрема змішана фаза співіснування феромагнетизму і незвичної надпровідності викликається спонтанною намагніченістю. Змішана фаза $€$ стійкою, в той час як інші надпровідні фази, що зазвичай існують у нетрадиційних надпровідниках, є або нестійкими, або, при певних значеннях параметрів теорії, метастабільними при відносно низьких температурах у вкрай вузькій області фазової діаграми. Фазовий перехід із нормальної фази у фазу співіснування $є$ переходом першого роду, в той час як фазовий перехід із феромагнітної фази у фазу співіснування може бути або першого або другого роду залежно від типу речовини. Куперівське спарювання і анізотропія кристалу є суттєвими для більш точного опису форми фазової діаграми і знімають виродження основного стану системи, проте вони не чинять значного впливу на області фазової стійкості i термодинамічні властивості відповідних фаз.

Ключові слова: надпровідність, феромагнетизм, фазова діаграма, профіль параметра впорядкуваня, анізотропія

PACS: 74.20.De, 74.20.Rp 